\title{
Design of a Hybrid Multi-Occupant Visitor Communication and Door Control System
}

\author{
A.O. Adeyemi-Ejeye, M. Mehdi, M.G Martini, N. Phillip, J. Orwell \\ Kingston University, London \\ \{a.adeyemi-ejeye,m.mehdi,m.martini,n.philip,james\}@kingston.ac.uk
}

\begin{abstract}
Door phone systems, allowing occupants of a building to communicate with visitors at the door, have evolved over the years, with the current advancements being a fully internet protocol (IP) based solution. In order to adopt newer IP based solutions, current analogue systems can be replaced, yet this may be costly and cumbersome, especially in a conventional multioccupant building. We therefore propose an architecture which supports current analogue door phone systems, and also provides IP based functionality. We have implemented the proposed architecture based on SIP, WebRTC and an IoT gateway system connected to the multi-occupant conventional video door phone system.

Index Terms-IoT, Door Entry, SIP, WebRTC.
\end{abstract}

\section{INTRODUCTION}

The Internet of things (IoT) provides possibilities for both communication and control of devices and appliances within buildings, through the use of user interactive control solutions [1]. With the increased capabilities and growing acceptance of IoT technology, it seems worthwhile to investigate how this technology can provide useful features for people using traditional door phone entry systems. A hybrid approach seems to be the most favourable, as it introduces an effective interface, and can still incorporate fail-safe characteristics of the analogue system, making it less expensive when compared to replacing the entire system.

In this paper, we propose a flexible and enhanced hybrid multi-occupant visitor communication door control system architecture which combines traditional video door phone services (analogue audio/video) and IP services, such as video and voice over IP (VoIP). A major limitation of conventional video door entry systems is that the occupant can only communicate with their visitor using a fixed video phone device, and this is obtainable on either a one-to-one or multi-occupant system. Although a solution has been provided in [2] for the legacy one-to-one system, which enables the visitor communicate using session initiation protocol (SIP) [3] and a home gateway, it is limited as it only extends the one-to-one system (one door phone to one residential dwelling), and therefore would not be suitable for multi-occupancy environment, where the door phone would be able to communicate $\mathrm{N}$ occupants of a building, either flats or offices.

To overcome the current limitations, we extend the contributions of [2], and thus propose a Hybrid Multi-Occupant Visitor Communication Service (HMVCS) architecture. The HMVCS is based on an IoT gateway (IoTG), Dual-Tone MultiFrequency (DTMF) [4], SIP and Web Real-Time Communi- cation (WebRTC) [5], making it flexible to deploy in both residential and office buildings.

\section{Hybrid Multi-OcCupant Visitor COMMUNiCATION SERVICE}

\section{A. Overall System}

The overall operation of the proposed system for the HMVCS can be seen in Fig. 1. It consists of a multi-occupant video door phone system, an IoTG and user devices such as tablets, PCs, mobile phones that can be connected either to the Internet or a cellular network.

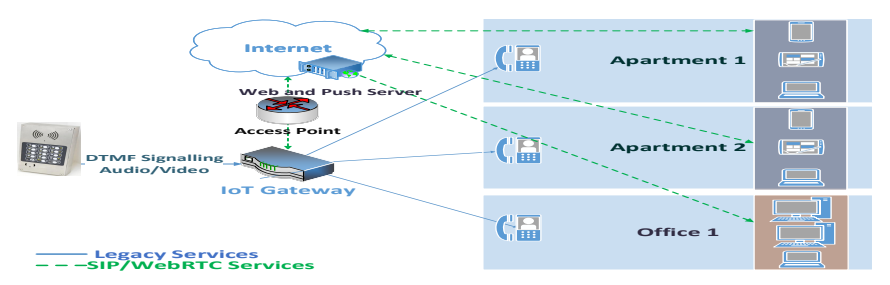

Fig. 1. The Hybrid Multi-Occupant Visitor communication System

\section{B. SIP, WebRTC and DTMF}

SIP is a major signaling protocol used in VoIP, with a major feature being name mapping that enables users to initiate or receive communications and services on different devices irrespective of their IP address and location via a uniform resource identifier (URI). Therefore, users can benefit from a 1-to-n scenario, with one address and many devices.

WebRTC is based on web technologies enabling real-time audio, video and data communication between web browsers. Unlike SIP, WebRTC uses Javascript Session Establishment Protocol (JSEP) [6], to specify the generic need for information transfer rather than a particular signalling model, so that both ends are aware of how communication will be conducted. While DTMF is a method used for transmitting control signals over a communication channel [7], and can be found in many applications such as robotics, banking and legacy door entry systems.

Therefore, the IoTG is defined as the HMVCS entity, which performs both SIP and WebRTC server functionalities such Session Traversal Utilities for NAT (STUN). In addition, as a user agent (UA) for both SIP and WebRTC sessions, it has an audio/video codec and a software-based analogue-digital DTMF signal converter, which triggers door phone device 
events. The IoTG is connected to the internet, making it possible for it to communicate with those who have authorized credentials, according to their preferences or via a terminal anytime or anywhere. These preferences are either based on registered devices or address credentials. For example to establish a SIP based call, the Door Phone Agent (DPA) would need to have the callee's SIP URI in its address book, which can either be based on a private branch exchange or a telephone number, while the WebRTC calls can be made using any compatible Internet browser.

\section{PRototype Implementation}

The Physical interface of the existing traditional MultiOccupant door phone device was connected to the IoTG, which controls the door phone system and transmits video and audio signal received from the door phone device to the user terminal and vise versa. In our architecture, JSEP and SIP were used to establish both WebRTC and SIP calls respectively, while RTP is used to transmit and receive both digital multimedia and DTMF signals between the gateway and the user terminal. In addition, the gateway also initiates the push notifications to notify the user to make a WebRTC call. Fig. 2 shows the message flow for the HMVCS, while Fig. 3 shows the terminal of the IoTG when connected to the DPA. The HMVCS UA, SIP-based application or mobile

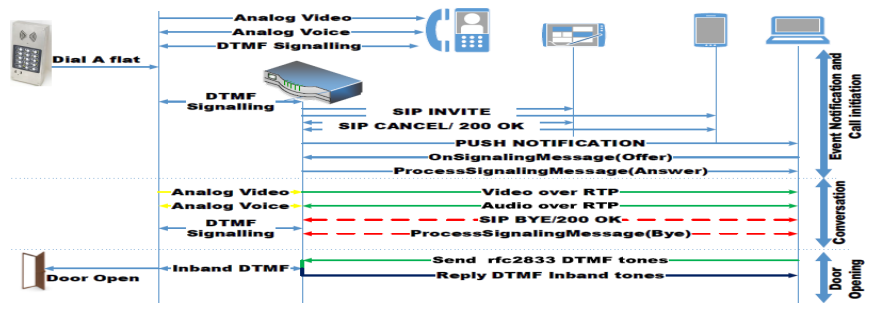

Fig. 2. Message Flow for the HMVCS

phone can be initiated anywhere, and does need to register with its providers respective register server. The IoTG would need to store either the SIP address, telephone number or Push notification identity (WebRTC).

1) Event Notification and Call Initiation Phase: When the visitor dials the flat/office number, the IoTG decodes the DTMF tones in real-time and then checks its address book for the event to trigger. For a SIP based call, the SIP INVITE message is sent to the registered user terminals for the flat dialed. Each device receives a door ring event and can accept the call on any of the devices. For the WebRTC call session, the gateway push client sends a notifcation to the User, and also initialises the chat-room. On receving the notification, the User can connect the IoTG using the web link provided.

2) Conversation Phase: When both IoTG and the user Terminal are connected, there is multimedia exchange, where audio communication is bidirectional, while the video is being transmitted from the gateway using the RTP protocol. If the user wants the call terminated, the user clicks on "CLOSE/END" button, and the "BYE" message is sent from the HMVCS UA to the IoTG, which relies "OK".
3) Door Opening Phase: The user confirms the visitor and opens the door, using RFC2833 [8] DTMF tones which are transmitted to the IoTG. In turn the IoTG uses its DTMF interpreter module to generate the analogue "DOOR OPEN" signal. This is the same procedure as on a SIP/WebRTC call session. In response, the DPA sends the end DTMF signal to the IoTG which in turn sends the "BYE" message to the HMVCS UA. The HMVCS UA replies "OK", and finally the multimedia session between them is released.

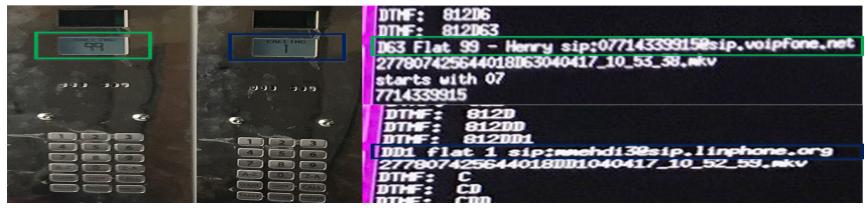

Fig. 3. A Multi-Occupant door phone device connected to the IoTG

\section{CONCLUSION}

We have proposed and implemented a Hybrid MultiOccupant Visitor Communication System as the architecture in which an occupant in a multi-occupation building such as an office/a block of flats can confirm and converse with a visitor anywhere and anytime, while still maintaining its legacy door phone system. The occupant can communicate with a wide range of options such as SIP phone software such as Linphone [9], telephones, and web browsers. At this time, we are planning to extend our architecture to use DTMF-over-RTP for self access control.

\section{ACKNOWLEGDEMENTS}

This research described here is supported by the Knowlegde Transfer Partnership under KTP Partnership Number: KTP010129. We would also like to thank The Entryphone Company Ltd. for their support.

\section{REFERENCES}

[1] L. Atzori, A. Iera, and G. Morabito, "The internet of things: A survey," Computer networks, vol. 54, no. 15, pp. 2787-2805, 2010.

[2] O. Yeon-Joo, P. Eui-Hyun, and P. Kwang-Roh, "Design of a SIPbased real-time visitor conversation and door control architecture using a home gateway," in 2006 IEEE International Conference on Consumer Electronics, Jan 2006, pp. 187-188.

[3] J. Rosenberg, H. Schulzrinne, G. Camarillo, A. Johnston, J. Peterson, R. Sparks, M. Handley, and E. Schooler, "SIP: session initiation protocol," Tech. Rep., 2002.

[4] N. B. Cox and E. L. Froese, "Multi-frequency signal detector and classifier," Oct. 4 1994, US Patent 5,353,346.

[5] A. Bergkvist, D. C. Burnett, C. Jennings, and A. Narayanan, "Webrtc 1.0: Real-time communication between browsers," Working draft, W3C, vol. $91,13 / 03 / 2017$.

[6] E. Rescorla, "Javascript session establishment protocol draft-ietf-rtcwebjsep-07," IETF Internet Draft, 2014.

[7] Y. C. Cho and J. W. Jeon, "Remote robot control system based on DTMF of mobile phone," in Industrial Informatics, 2008. INDIN 2008. 6th IEEE International Conference on. IEEE, 2008, pp. 1441-1446.

[8] H. Schulzrinne and S. Petrack, "RFC 2833: RTP payload for DTMF digits, telephony tones and telephony signals," IETF Network Working Group Request for Comments, vol. 2833, 2000.

[9] B. Communications, "Linphone: Open source voip project," 2001 [Online]. Available: http://www.linphone.org/ 